\title{
Objectively characterizing Huntington's disease using a novel upper limb dexterity test
}

\author{
Samuel Woodgate ${ }^{1,2}$ (D) Philippa Morgan-Jones ${ }^{1,3}$ (C) Susanne Clinch ${ }^{4} \cdot$ Cheney Drew $^{3} \cdot$ Rebecca Playle $^{3}$. \\ Mohamed Bennasar ${ }^{5}$. Yulia Hicks ${ }^{1}$. Catherine Holt ${ }^{1}\left[\right.$ - Anne-Catherine Bachoud-Lévi ${ }^{6,13}$ - Renaud Massart ${ }^{6,13}$. \\ David Craufurd $^{7,8} \cdot$ Nigel Kirby $^{3} \cdot$ Katy Hamana $^{9} \cdot$ Robin Schubert $^{10} \cdot$ Ralf Reilmann $^{10,11,12} \cdot$ Anne Rosser $^{2,4}$ (i) \\ Monica Busse ${ }^{3}$ (1)
}

Received: 18 August 2020 / Revised: 14 December 2020 / Accepted: 15 December 2020 / Published online: 8 February 2021

(c) The Author(s) 2021

\begin{abstract}
Background The Clinch Token Transfer Test (C3t) is a bi-manual coin transfer task that incorporates cognitive tasks to add complexity. This study explored the concurrent and convergent validity of the $\mathrm{C} 3 \mathrm{t}$ as a simple, objective assessment of impairment that is reflective of disease severity in Huntington's, that is not reliant on clinical expertise for administration. Methods One-hundred-and-five participants presenting with pre-manifest $(n=16)$ or manifest (TFC-Stage- $1 n=39$; TFCStage- $2 n=43$; TFC-Stage- $3 n=7$ ) Huntington's disease completed the Unified Huntington's Disease Rating Scale and the $\mathrm{C} 3 \mathrm{t}$ at baseline. Of these, thirty-three were followed up after 12 months. Regression was used to estimate baseline individual and composite clinical scores (including cognitive, motor, and functional ability) using baseline C3t scores. Correlations between C3t and clinical scores were assessed using Spearman's R and visually inspected in relation to disease severity using scatterplots. Effect size over 12 months provided an indication of longitudinal behaviour of the $\mathrm{C} 3 \mathrm{t}$ in relation to clinical measures.

Results Baseline C3t scores predicted baseline clinical scores to within 9-13\% accuracy, being associated with individual and composite clinical scores. Changes in C3t scores over 12 months were small $(\Omega \leq 0.15)$ and mirrored the change in clinical scores.

Conclusion The $\mathrm{C} 3 \mathrm{t}$ demonstrates promise as a simple, easy to administer, objective outcome measure capable of predicting impairment that is reflective of Huntington's disease severity and offers a viable solution to support remote clinical monitoring. It may also offer utility as a screening tool for recruitment to clinical trials given preliminary indications of association with the prognostic index normed for Huntington's disease.
\end{abstract}

Keywords Huntington's disease $\cdot$ Outcome measure $\cdot$ Upper-limb function $\cdot$ Convergent validity $\cdot$ Regression

Samuel Woodgate and Philippa Morgan-Jones shared joint first authorship.

Supplementary Information The online version contains supplementary material available at https://doi.org/10.1007/s0041 5-020-10375-8.

Monica Busse

busseme@cardiff.ac.uk

Extended author information available on the last page of the article

\section{Introduction}

Huntington's disease (HD) is an autosomal dominant neurodegenerative disorder that affects $6-13 / 100,000$ in the general population [1]. HD is caused by the expansion of a cytosine-adenine-guanine (CAG) triplet repeat expansion on the Huntingtin gene which leads to pathological symptoms once the repeats reach thirty-six or more.

HD is characterized by a complex presentation of motor, cognitive, behavioral and functional impairments [2], and results in a progressive decline in quality of life over 15-20 years, ultimately leading to early death. Whilst there is currently no recognized cure, substantial progress is being made in disease modifying therapeutic interventions, with 
clinical trials underway [3]. With a real opportunity to slow functional decline on the horizon, there is an ever-increasing urgency for clinical endpoints that can truly determine the influence an intervention has on clinical progression.

At present, clinical trials typically rely on clinicianreported assessments, with the Unified Huntington's Disease Rating Scale (UHDRS) routinely used to assess clinical performance and capacity in HD [4]. Individual components within the UHDRS facilitate the assessment of motor function, cognitive function, behavior and functional capacity $[5,6]$. Whilst assessments of individual domains have furthered understanding of specific aspects of disease progression [7-9], pre-existing scales are thought to lack sensitivity to change and are prone to ceiling affects in pre-manifest and early HD [10]. There also remains a distinct lack of validated clinical tools capable of assessing upper limb function in HD [11]. Such assessments are needed when seeking to understand the real life implications that a movement disorder such as HD has on activities of daily living [12]. Furthermore, the current COVID-19 pandemic has highlighted a heavy reliance on face to face assessments and a real need for robust tools that allow continued remote monitoring of symptoms when face to face clinic visits prove challenging. Subsequently, there is a recognized need for more granular and novel assessments of clinical symptoms, that can be performed in the clinic and home environments, to better evaluate the influence of novel interventions in HD.

The Moneybox test (herein referred to as the Clinch Token Transfer Test (C3t)) is a bi-manual upper limb token transfer and manipulation performance assessment, with a view to providing an ecologically valid functional assessment underpinned by sound biological rationale for people with basal ganglia dysfunction [13]. The $\mathrm{C} 3 \mathrm{t}$ has the benefits of being quick to perform and objective in nature, with no reliance on clinical expertise to administer and thus real potential to deliver a remote solution, for monitoring clinical symptoms at scale. This in turn would increase the depth of information available to researchers to help identify and understand subtle changes in disease progression.

Early validation work found the $\mathrm{C} 3 \mathrm{t}$ to be sensitive to HD disease stage and correlated to the components of the UHDRS and health related quality of life [13]. An instrumented version of the test, involving accelerometer data from devices worn on each wrist and the sternum, has also successfully discriminated between healthy and HD participants, with C3t derived scores highly correlated to upperbody clinician rated motor symptoms [14]. Whilst extremely promising, investigations are now required into how well the $\mathrm{C} 3 \mathrm{t}$ can predict clinical symptoms to further evaluate its utility as an outcome measure. Additionally, work needs to be extended to investigate how well the $\mathrm{C} 3 \mathrm{t}$ scores correlate to composite clinical scores given the multifaceted presentation of HD symptoms. Two notable composite scores requiring investigation are i) the composite UHDRS (cUHDRS) which is thought to be a stronger global indicator of disease progression, capturing the changes occurring simultaneously across the individual symptom domains in HD [15] and ii) the Prognostic Index normed for HD (PIN ${ }_{\mathrm{HD}}$ ) [16] which has been developed to determine projected disease progression.

This study subsequently explored the concurrent and convergent validity of the C3t as a simple, objective assessment of impairment that is reflective of disease severity in Huntington's.

\section{Methods}

\section{Recruitment and governance}

Data presented here were drawn from datasets across four different studies-PACE-HD, CAPIT-HD2, TRIDENT and Developing Clinical Applications for a Novel Multi-Task Functional Assessment: The Clinch Token Transfer Test (referred to here as NOVELFA-C3T).

PACE-HD (Clinical trials registration: NCT03344601) is a multi-center trial with sites in Germany, Spain, and USA, where all participants were also concurrently participating in Enroll-HD. As PACE-HD is an ongoing intervention study, only baseline data were included.

CAPIT-HD2 was a multi-center study with data collected across 4 different sites in Europe (Cardiff and Manchester, UK; Créteil Paris, France; Muenster, Germany). In the Cardiff, Manchester and Muenster sites, participants were recruited from those currently participating in the global Enroll-HD study (https://www.enroll-hd.org/). In Créteil, participants were recruited from those currently enrolled in the Predictive Biomarkers for Huntington's disease study (Clinical trials registration: NCT01412125). Participants completed a battery of assessments during a baseline visit and were then invited to return for 1-month and 12-month visits to control for a retest effect [17]. Data collated at the 1-month timepoint involved a reduced battery of assessments (i.e., did not reflect the minimum dataset required for analyses) and was thus omitted from the analysis reported here.

TRIDENT and NOVELFA-C3T are both single-site studies based in Cardiff. Participants were recruited from those currently participating in the global Enroll-HD study. They were invited to attend a single baseline visit to complete the requisite test battery. Ethical approval for all studies was granted by Health and Care Research Wales (CAPITHD2 REC: 17/WA/0014, TRIDENT REC: 18/WA/0182, NOVELFA-C3T REC: 17/WA/0014).

All participants included in the studies were 18 or more years of age, with genetically confirmed HD and the capacity to provide informed consent. Diagnosis of HD was categorized into one of four disease stages at each visit 
(Pre-manifest, Diagnostic Confidence Interval (DCI) $\leq 3$; Stage 1, Total Functional Capacity $(\mathrm{TFC})=11-13$ and $\mathrm{DCI}=4$; Stage 2, TFC =7-10; Stage 3, TFC $=4-6$ ) [15]. The CAG Age Product score (CAP) [18] was calculated using age and $\mathrm{CAG}$ repeat length to estimate disease impact for demographic purposes [19] (Eq. 1).

$\mathrm{CAP}=$ Age $* \frac{\mathrm{CAG}-30}{6.49}$

\section{Assessments and outcome measures}

All participants $(n=105)$ performed the $\mathrm{C} 3 \mathrm{t}$ or a prior version of the task (Moneybox Test (MBT)) at the baseline visit with a subset repeating this at 12 months $(n=33)$. Both versions of the test involve the performance of six (C3t) or five (MTB) tasks, with this study focusing on two of the tasks performed identically across test versions- the Baseline Transfer Task (BTT) and Complex Transfer Task (CTT) which are described in full in the C3t manual (Supplementary Material 1). In both BTT and CTT tasks, participants picked up a token one at a time with their non-dominant hand, transferred it to their dominant hand and placed it into a slotted box. During the BTT participants transferred eight blank tokens in order of physical size (largest to smallest). During the CTT a mild cognitive load is added by asking participants to transfer a different set of tokens in order of the number printed on them (highest to lowest). In both tasks the primary measure recorded is the time taken to complete the task (i.e., transfer all tokens successfully).

Participants also completed the Unified Huntington's Disease Rating Scale (UHDRS), with scores produced to assess the symptom domains of motor function (Total Motor Score (TMS)) and cognition (Symbol Digit Modalities Test (SDMT) and Stroop Word Reading Test (SWRT)) along with capacity (Total Functional Capacity (TFC)). The composite UHDRS (cUHDRS) [15] was used to provide a global indicator of HD disease progression (Eq. 2).

$$
\begin{aligned}
\mathrm{cUHDRS}= & {\left[\left(\frac{\mathrm{TFC}-10.4}{1.9}\right)-\left(\frac{\mathrm{TMS}-29.7}{14.9}\right)\right.} \\
& \left.+\left(\frac{\mathrm{SDMT}-28.4}{11.3}\right)+\left(\frac{\mathrm{SWR}-66.1}{20.1}\right)\right]+10
\end{aligned}
$$

Scores on the select UHDRS assessments (TMS, SDMT, SWRT, TFC) and the cUHDRS were used as criterion clinical measures to assess the convergent and concurrent validity of the $\mathrm{C} 3 \mathrm{t}$.

The prognostic index normed for HD (PIN $\left.{ }_{\mathrm{HD}}\right)$ [16] was calculated (Eq. 3) to determine projected disease progression in the pre-manifest subgroup, with higher scores indicating greater risk of motor diagnosis. A PIN ${ }_{\mathrm{HD}}$ of less than 0 indicates greater than 50\% 10-year survival, whilst a PIN $\mathrm{HD}_{\mathrm{H}}$ of greater than 0 indicated less than 50\% 10-year survival.

$\mathrm{PIN}_{\mathrm{HD}}=\frac{\mathrm{PI}_{\mathrm{HD}}-883}{1044}$

where

$\mathrm{PI}_{\mathrm{HD}}=51 \times \mathrm{TMS}+(-34) \times \mathrm{SDMT}+7 \times \mathrm{Age} \times(\mathrm{CAG}-34)$

All data were stored in a SQL database using Python (v3.7) and subsequently analyzed using SciPy (v1.3.0) [20].

\section{Data analysis}

The C3t time taken BTT and CTT scores were assessed for normality using multiple statistical tests (Shapiro-Wilks Test, D'Agostino K-Squared Test and Anderson-Darling Test) and visually inspected using histograms and Q-Q plots. Given their non-normal distribution, non-parametric data analysis methods were employed.

Least squares regression was performed to determine whether HD disease severity, as measured by the UHDRS (cUHDRS, TMS, SMDT and SWRT), could be predicted using C3t scores (BTT and CTT). The coefficients from a LASSO regression (see Supplementary Material 2) confirmed that data collected across sites and using the two different versions of the $\mathrm{C} 3 \mathrm{t}$ could be pooled as both were found to have negligible effects on the regression model. Scatterplots of C3t time scores (BTT and CTT) plotted against the TMS, SDMT, SWRT and cUHDRS revealed a non-linear relationship and as such a polynomial regression (degree of 2 ) was performed to optimize the predictive model. The TFC was not predicted due to a lack of any discernable pattern with the $\mathrm{C} 3 \mathrm{t}$ scores (which was confirmed via a scatterplot of C3t time scores (BTT and CTT) plotted against TFC). The PIN $_{\mathrm{HD}}$ was also not predicted due to insufficient sample size as this could only be measured for the pre-manifest subgroup $(n=16)$. Only data collected during the baseline visit were used in the regression models. To ensure robust results, repeated $k$-fold cross-validation was used. Cross-validation is a common method used in machine learning to avoid overfitting statistical models to datasets, helping to ensure robust, generalizable results. $K$-fold cross-validation splits the dataset into $k$ partitions, withholding one partition and training/constructing a regression model using the remaining $k-1$ partitions. The quality of the trained regression model is then assessed using the withheld partition. This process is conducted $\mathrm{k}$ times, with each partition taking a turn at being withheld and used to assess a regression model trained using the remaining $k-1$ partitions. In repeated $\mathrm{k}$-fold crossvalidation this process is then repeated by randomly shuffling the original dataset. Doing so is designed to ensure the 


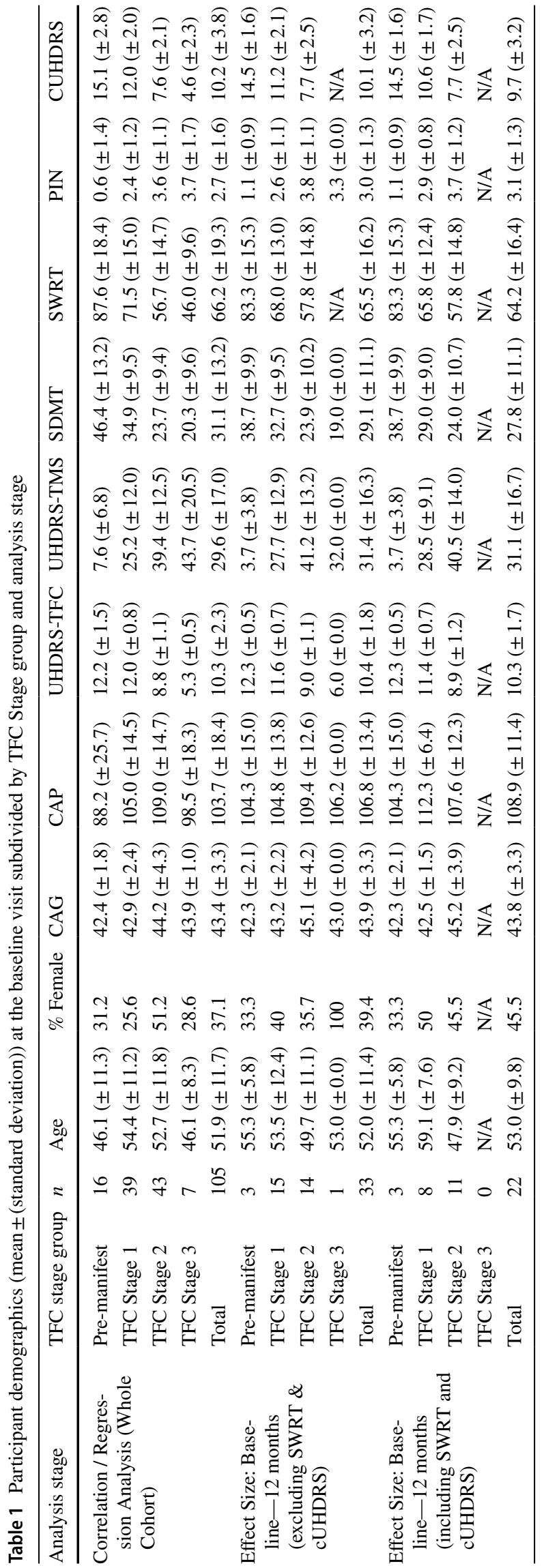

internal structure of the original dataset has not, by chance, influenced the results of the models.

In this study four folds and ten repeats were used $(k=4$, repeats $=10$ ), resulting in 40 models constructed overall. During each cross-validation fold, the ratio of TFC Stages present in the entire dataset was maintained in the training and testing sets. The mean absolute error (MAE) and the normalized MAE were used to assess model quality for each of the 40 models generated during the cross-validation process (Eq. 4). The mean MAE and normalized MAE across the 40 models are reported. The MAE quantifies on average how far off from the actual value (regardless of direction) a model is, across a population when predicting a dependent variable (in this case the UHDRS scores). The normalized MAE was utilized to allow comparison across outcomes with varying ranges:

MAE $=\frac{\sum_{i=1}^{n}\left|y_{i}-x_{i}\right|}{n}$

where $y_{i}$ is the actual clinical score, $x_{i}$ is the predicted score and $\mathrm{n}$ is the sample size.

The strength and direction of association between each clinical score and the $\mathrm{C} 3 \mathrm{t}$ scores was measured using Spearman's $R$ correlation coefficient. A-priori statistical level of significance was set to $p \leq 0.05$.

Scatterplots are reported for each clinical score in relation to the BTT and CTT scores, with a key denoting disease stage for each participant. These were visually inspected to evaluate the sensitivity of the $\mathrm{C} 3 \mathrm{t}$ to measure clinical impairment across the spectrum of disease state. PIN $\mathrm{HD}_{\mathrm{HD}}$ was plotted in a similar manner against the BTT and CTT scores to investigate how sensitive the $\mathrm{C} 3 \mathrm{t}$ scores may be to overall risk of motor diagnosis and probability of 10 -year survival. With the PIN $_{\mathrm{HD}}$ designed to estimate progression levels in pre-diagnosis HD, this was only performed on the pre-manifest sub-group.

Baseline to 12-month changes in C3t scores were assessed using effect size and compared to clinical scores using data from all participants, where follow-up data were available ( $n=33$, with 11 lacking a SWRT score thus reducing sample size to 22 when measuring change in SWRT and cUHDRS). Effect size was calculated using a nonparametric analog of Cohen's $D$, omega $(\Omega)$, where $\Omega=0$ indicates no effect and values of $\pm 0.1, \pm 0.3$ and \pm 0.4 correspond to the descriptors used for Cohen's $D$ indicating low, medium and large effect sizes, respectively [21]. Unlike Cohen's $D, \Omega$ is directional and as such for the sake of simplicity the absolute value of $\Omega$ is reported throughout. 
Table 2 Regression results for C3t scores and clinical measures at baseline

\begin{tabular}{|c|c|c|c|c|c|}
\hline C3t score & Clinical score & Spearman's $R$ & $\begin{array}{l}\text { Mean absolute error } \\
( \pm \text { SD })\end{array}$ & $\begin{array}{l}\text { Normalised mean } \\
\text { absolute error }\end{array}$ & $\begin{array}{l}\text { Cohort max } \\
\text { clinical value }\end{array}$ \\
\hline \multirow[t]{4}{*}{ Baseline transfer task time taken } & cUHDRS & $-0.69 * *$ & $2.23( \pm 0.33)$ & $11.0 \%( \pm 2.0 \%)$ & 19.89 \\
\hline & TMS & $0.67 * *$ & $9.88( \pm 1.43)$ & $13.0 \%( \pm 2.0 \%)$ & 78 \\
\hline & SMDT & $-0.63 * *$ & $8.14( \pm 1.08)$ & $11.0 \%( \pm 2.0 \%)$ & 72 \\
\hline & SWRT & $-0.62 * *$ & $12.05( \pm 1.8)$ & $9.0 \%( \pm 1.0 \%)$ & 134 \\
\hline \multirow[t]{4}{*}{ Complex transfer task time taken } & cUHDRS & $-0.7 * *$ & $2.11( \pm 0.3)$ & $11.0 \%( \pm 1.0 \%)$ & 19.89 \\
\hline & TMS & $0.69 * *$ & $9.4( \pm 1.18)$ & $12.0 \%( \pm 2.0 \%)$ & 78 \\
\hline & SMDT & $-0.64 * *$ & $7.63( \pm 1.15)$ & $11.0 \%( \pm 2.0 \%)$ & 72 \\
\hline & SWRT & $-0.64 * *$ & $12.02( \pm 1.47)$ & $9.0 \%( \pm 1.0 \%)$ & 134 \\
\hline
\end{tabular}

Spearman's $R$ (** indicates $p<0.001$ ), its corresponding $p$-value, the Mean Absolute Error (MAE) and Normalised MAE are reported. Spearman's $R$ is calculated across the entire baseline dataset. The MAE and Normalised MAE are the mean results from repeated, $k$-fold cross-validated models $(k=4$, repeats $=100)$ stratified across TFC stages. MAE and Normalised MAE results are the mean $( \pm$ standard deviation) across all folds. Cohort max is defined as the maximum value for each clinical measure in the dataset

\section{Results}

\section{Participants}

One-hundred-and-five gene-positive participants were recruited at the baseline visit across all studies and sites (see Table 1, with the number of participants recruited across each sub-study and disease stage reported in Supplementary Material 3). Thirty-three participants also attended the 12-month follow-up visit.

\section{C3t scores in relation to UHDRS clinical scores}

C3t time scores and each of the four UHDRS measures during the baseline were highly and significantly associated $(p<0.001)$ with one another (see Table 2$)$ with the strongest correlation identified between the cUHDRS and the CTT time scores (CTT $r=-0.7$ ). A positive association was found between time taken to perform both $\mathrm{C} 3 \mathrm{t}$ tasks and TMS, where BTT and CTT time increased as TMS score increased. In contrast, negative associations were found for the cUHDRS, SMDT and SWRT, where time taken to perform BTT and CTT increased as clinical scores decreased. Scatterplots plotting each clinical score under analysis against the BTT and CTT C3t scores are presented in Fig. 1 to visually represent these associations (with a key denoting disease stage for each participant to aid contextualization). No further investigation of relationships between $\mathrm{C} 3 \mathrm{t}$ and TFC were indicated given the lack of any association between C3t scores and the TFC (see Fig. 2). When estimating baseline clinical scores using baseline $\mathrm{C} 3 \mathrm{t}$ scores, normalized mean absolute error ranged from at best $9 \%$ and at worst $13 \%$ (see Table 2).

\section{C3t scores in relation to predicted 10 -year survival rate and motor diagnosis}

A positive correlation was found between timed taken to perform both C3t tasks and $\mathrm{PIN}_{\mathrm{HD}}$ in the pre-manifest subgroup (BTT $r=0.83, p<0.001$; CTT $r=0.76, P<0.05$ ), where BTT and CTT increased as PIN $_{\mathrm{HD}}$ score increased (see Fig. 3).

\section{Assessing the 12-month behavior of the C3t}

Small changes in C3t scores were reported for baseline to 12 -month changes $(\Omega \leq 0.15)$ which mirrored the small change reported in all clinical scores (see Table 3 ).

\section{Discussion}

The $\mathrm{C} 3 \mathrm{t}$ is a quick, easy to administer objective assessment that is associated with HD disease status. Previous work has shown that the time to perform C3t tasks is related to increasing disease manifestation [13]. Here we have re-confirmed these findings on a significantly larger cohort with representation across a broader spectrum of disease. Additionally, we have shown that the BTT and CTT task times can be used to predict gold-standard motor, cognitive and composite clinical measures with a high-degree of accuracy.

In the original development of the $\mathrm{C} 3 \mathrm{t}$, it was proposed that the dual task paradigm would elicit motor impairment perhaps not seen in simple or single task conditions [22]. Yet, it appears that time taken to perform the simple task (BTT) and dual task (CTT) are correlated with both motor and cognitive clinical outcomes (i.e., increased time to complete the C3t tasks may be as a result of cognitive or motor deficits or a combination of both). Whilst it is possible that individuals with HD could exhibit differing prioritization 
Journal of Neurology (2021) 268:2550-2559

2555
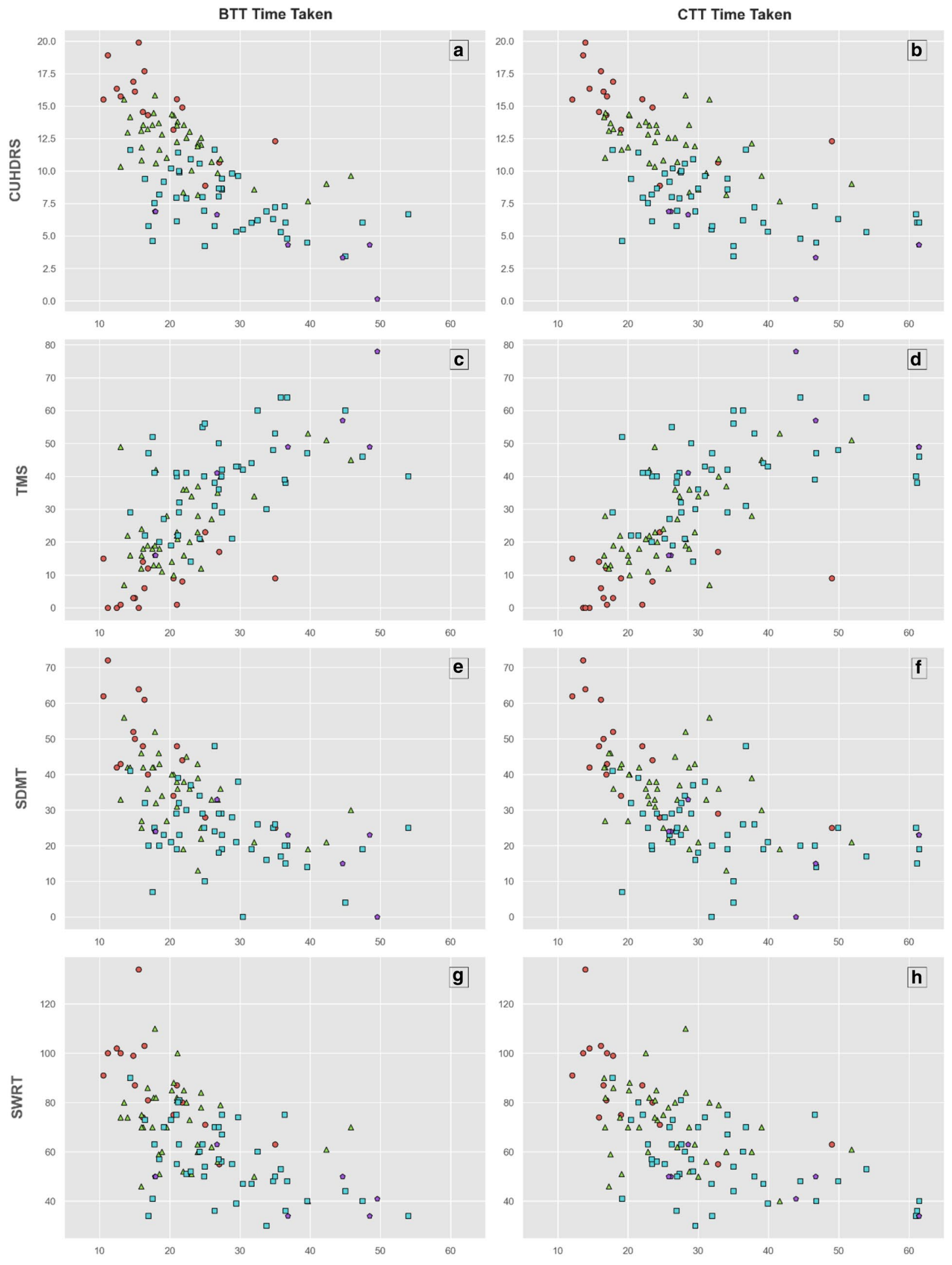

- Pre-manifest $\triangle$ TFC Stage 1 a TFC Stage 2 - TFC Stage 3

Springer 
४Fig. 1 Time taken to perform the Baseline Transfer Task (BTT) and Complex Transfer Task (CTT) plotted against the Composite Unified Huntington's Disease Rating Scale (cUHDRS), Total Motor Score (UHDRS-TMS), Symbol Digit Modalities Test Number Correct (SDMT) and Stroop Word Reading Test (SWRT). Participant disease stage is denoted by the key

strategies based on cognitive and motor ability, previous studies have found that the majority of participants exhibited mutual interference or prioritized motor over cognitive activities [23]. With the BTT and CTT analyses demonstrating comparable findings, we suggest that there is limited added value in performing both the BTT and CTT when seeking to estimate global clinical progression using the time taken to perform a given task. The utility of each task may, however, lie beyond these simple temporal measures and requires further investigation.

The notion of multiple domains influencing clinical outcomes has been identified by Schobel [15] who recommended the use of a global measure of clinical disease progression in the cUHDRS given the multifaceted presentation of HD. As a composite product of the UHDRS, the cUHDRS combines cognitive, motor, and global functional symptom domains, and has been found to have enhanced sensitivity to clinical change in early symptomatic HD [9]. The C3t timing measures were highly associated and predicted the cUHDRS to within $11 \%$ of the actual score.

$\mathrm{C} 3 \mathrm{t}$ scores were found to be associated to $\mathrm{PIN}_{\mathrm{HD}}$ in premanifest individuals, with a trend for increased time to perform the $\mathrm{C} 3 \mathrm{t}$ in people with higher $\mathrm{PIN}_{\mathrm{HD}}$ scores. Thus, it appears that the $\mathrm{C} 3 \mathrm{t}$ scores are capable of measuring symptoms in relation to predicted 10 -year survival rate and motor diagnosis. This in part would appear to be linked to the role that both the TMS and SDMT play in the calculation of $\mathrm{PIN}_{\mathrm{HD}}$ as these scores have independently been found to be associated with the C3t. Given the proposed utility of the PIN $_{\mathrm{HD}}$ to assist the identification of suitable participants into HD clinical trials [16], the $\mathrm{C} 3 \mathrm{t}$ shows promise as a recruitment screening tool that could lead to improved efficiency in clinical trials. With pre-manifest participants representing only a relatively small subgroup of the current study cohort, further investigation is, however, needed involving a larger pre-manifest cohort.

No association between the $\mathrm{C} 3 \mathrm{t}$ scores and function as measured by TFC was found. TFC is routinely used to define the stages of disease severity. It provides a standardized scale to assess capacity to work, deal with personal finances, perform domestic chores and activities of daily living and selfcare tasks. As such, many factors contribute to functional decline that is reflected by TFC scores. In contrast, the C3t is designed to primarily assess motor symptoms and has the potential to be impacted by cognitive impairment (e.g., psychomotor slowing and attentional deficits). As such, whilst some relationship might be expected, the two assessments are focused on different measurement domains.

Small changes in C3t scores over a 12-month period were in line with those seen in clinical outcomes over the same duration. Whilst greater changes in UHDRS over 12 months have been reported previously [15, 24], progression of clinical symptoms can be highly varied within the cohort under investigation. The ability to mirror changes in clinical outcomes is positive but further investigation is needed to ensure this is replicated when larger clinical progression is present. Investigations into the short-term stability of the $\mathrm{C} 3 \mathrm{t}$ when clinical symptoms remain stable is also warranted and will be the focus of future work.

A recognized limitation of this study is the under representation of later stage and pre-manifest participants. Surprisingly, later stage participants (Stage 3) were younger than those in earlier disease stages. We believe this is most likely a chance finding given the small numbers of late stage participants in our cohort $(n=7)$. Future work should focus on larger sample sizes and in particular the representation of both pre-manifest and later stage participants. Larger sample sizes per sub-group will allow further analysis to establish whether the predictive performance of the $\mathrm{C} 3 \mathrm{t}$ is stronger in a particular disease stage.

In conclusion, the time taken to perform the $\mathrm{C} 3 \mathrm{t}$ tasks is associated and reasonably predictive of HD disease status as assessed by the UHDRS. Being associated with PIN ${ }_{\mathrm{HD}}$ in pre-manifest participants, it may also offer utility as a screening tool for recruiting onto clinical trials. Furthermore, the $\mathrm{C} 3 \mathrm{t}$ scores mirrored the small changes in clinical impairment over 12 months. This study supports the potential utility of the $\mathrm{C} 3 \mathrm{t}$ to objectively estimate global clinical symptom severity in HD. It requires minimal equipment, time, and clinical expertise to perform, thus offering a viable solution for remote monitoring of clinical impairment. To facilitate such monitoring, emphasis now needs to be placed on streamlining the way in which the C3t can be performed in the home setting and how the data is returned to the clinical team. With the ability of sensor integration, the $\mathrm{C} 3 \mathrm{t}$ also facilitates the collection of detailed upper limb movement during task performance [14]. Thus, enhancing its potential as a sensitive assessment of motor function in clinical trials. Given the limited sample size available for the longitudinal analysis, future studies should have a specific focus on assessing the psychometric properties of the C3t over time and across the spectrum of disease manifestation. 


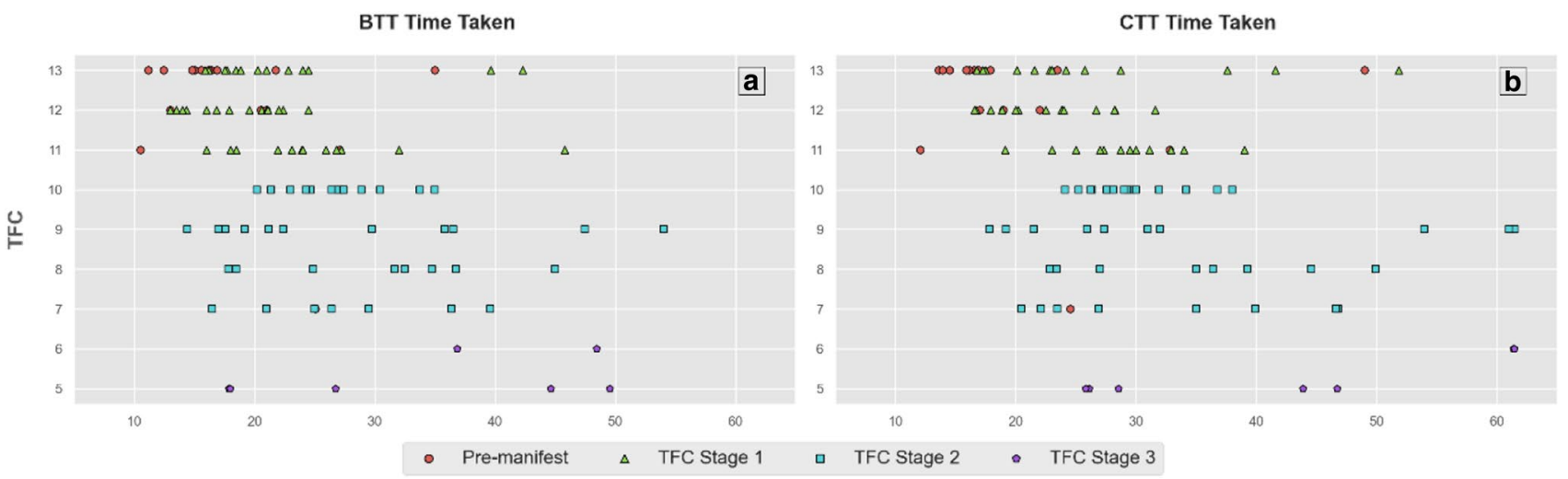

Fig. 2 Time taken to perform the Baseline Transfer Task (BTT) and Complex Transfer Task (CTT) plotted against the Total Functional Capacity (TFC) with disease stage of each participant denoted by the key

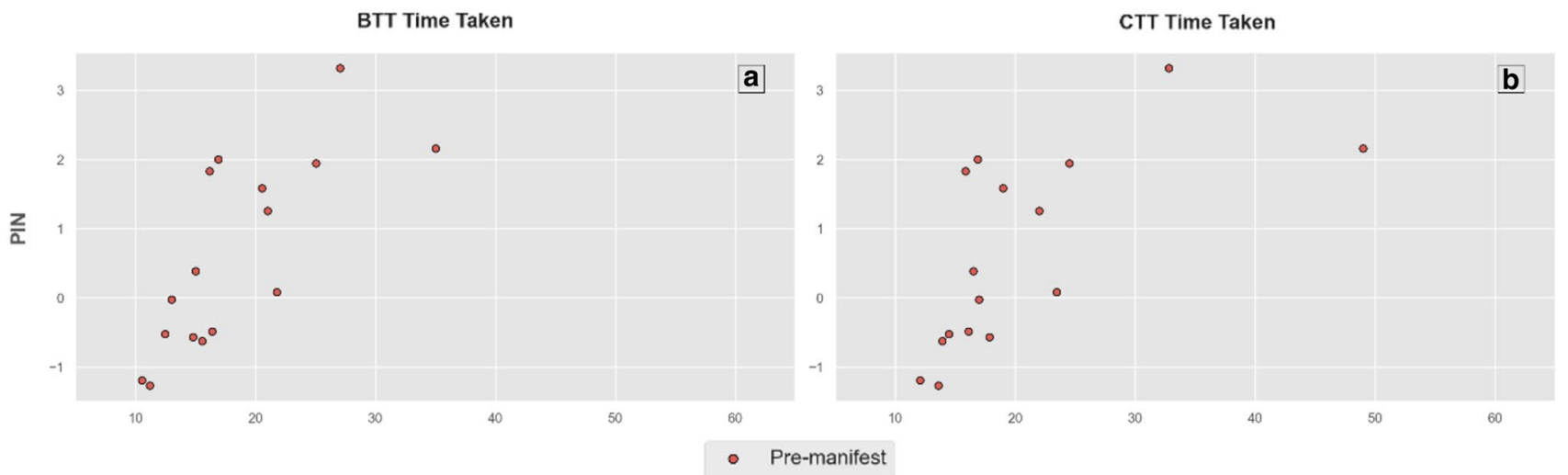

Fig. 3 Time taken to perform the Baseline Transfer Task (BTT) and Complex Transfer Task (CTT) plotted against Prognostic Index for HD $\left(\mathrm{PIN} \mathrm{HD}_{\mathrm{D}}\right)$ for pre-manifest participants

Table 3 Effect size, $\Omega$, (non-parametric analogue of Cohen's $D$ ) results for baseline to 12 months

\begin{tabular}{lc}
\hline & $\begin{array}{l}\text { Baseline to } \\
\text { 12-month effect } \\
\text { size }(\Omega)\end{array}$ \\
\hline Baseline Transfer Task (BTT) & -0.060 \\
Complex Transfer Task (CTT) & 0.100 \\
Total Motor Score (TMS) & -0.073 \\
Symbol Digit Modalities Test (SDMT) & 0.115 \\
Stroop Word Reading Test (SWRT) & 0.112 \\
Unified Huntington's Disease Rating Scale & 0.062 \\
$\quad$ (cUHDRS) & \\
\hline
\end{tabular}

Due to incomplete data, the sample size for SWRT and cUHDRS effect size was reduced from $n=33$ to $n=22$

Acknowledgements We acknowledge the REPAIR-HD consortium partners who served as scientific advisors to the clinical work packages through participation in workshops and critical discussions at consortium meetings and REPAIR-HD site staff who assisted in clinical data collection. We acknowledge additional contributors at partner sites namely, Jennifer Hamet (REPAIR-HD), L.Lemoine (REPAIR-HD) and K.Youssov (REPAIR-HD) assisted in delivery of the CAPIT-HD2 battery at National Centre of Reference for Huntington's Disease, Neurology department Henri Mondor Hospital, Creteil, France. Rebecca Cousins and Catherine Clengahan assisted in delivery of the CAPITHD2 battery and Laura Mills assisted in delivery of the TRIDENT battery, at the Cardiff Huntington's Disease Centre, School of Medicine, Cardiff University. Zara Skitt and Isobel McMillan assisted in delivery of the CAPIT-HD2 battery at the University of Manchester. We also acknowledge PACE-HD sites and staff in the United States (Re+Active Therapy and Wellness Centre/UCLA, Los Angeles; Teacher's College, Columbia University, New York), Germany (George Huntington Institute, Munster; University Hospital Ulm) and Spain (Merce de Deu de la Mare, Barcelona; Fundacion Jimenez Diaz, Madrid). The Centre for Trials Research receives funding from Health and Care Research Wales. The views expressed are those of the author/s and not necessarily those of Health and Care Research Wales or Welsh Government.

Funding MRC Confidence in Concept Scheme (Biomedical Catalyst) and Repair-HD funded from the European Union's Seventh Framework Programme for research, technological development and demonstration under grant agreement $n^{\circ} 602245$ (www.repair-hd-eu). 
Samuel Woodgate: Financial disclosures related to research covered in this article: Grants and Research: Medical Research Council, Cardiff University. Philippa Morgan-Jones: Financial disclosures related to research covered in this article: Grants and Research: None. Susanne Clinch: Financial disclosures related to research covered in this article: Grants and Research: Medical Research Council, European Framework. Cheney Drew: Financial disclosures related to research covered in this article: Grants and Research: Health and Care Research Wales (HCRW), Medical Research Council UK. Rebecca Playle: Financial disclosures related to research covered in this article: Grants and Research: Health and Care Research Wales (HCRW) and Medical Research Council. Mohamed Bennasar: Financial disclosures related to research covered in this article: Grants and Research: Medical Research Council. Yulia Hicks: Financial disclosures related to research covered in this article: Grants and Research: Medical Research Council. Catherine Holt: Financial disclosures related to research covered in this article: Grants and Research: Medical Research Council. Anne-Catherine Bachoud-Lévi Financial disclosures related to research covered in this article: Grants and Research: No financial disclosure to report. Renaud Massart: Financial disclosures related to research covered in this article: Grants and Research: No financial disclosure to report. David Craufurd: Financial disclosures related to research covered in this article: Grants and Research: European Framework funding. Nigel Kirby: Financial disclosures related to research covered in this article: Grants and Research: None. Katy Hamana: Financial disclosures related to research covered in this article: Grants and Research: None. Robin Schubert: Financial disclosures related to research covered in this article: Grants and Research: All compensations payed to the George-Huntington-Institute: the European Union's 7th Framework Program (EU-FP7) REPAIR-HD consortium. Ralf Reilmann: Financial disclosures related to research covered in this article: Grants and Research: All compensations payed to the GeorgeHuntington-Institute: the European Union's 7th Framework Program (EU-FP7) REPAIR-HD consortium. Anne Rosser. Financial disclosures related to research covered in this article: Grants and Research: European Framework funding, Medical Research Council UK. Monica Busse: Financial disclosures related to research covered in this article: Grants and Research: European Framework funding, Health and Care Research Wales (HCRW), Medical Research Council UK.

\section{Compliance with ethical standards}

Conflicts of interest The authors declare that they have no conflict of interest.

Open Access This article is licensed under a Creative Commons Attribution 4.0 International License, which permits use, sharing, adaptation, distribution and reproduction in any medium or format, as long as you give appropriate credit to the original author(s) and the source, provide a link to the Creative Commons licence, and indicate if changes were made. The images or other third party material in this article are included in the article's Creative Commons licence, unless indicated otherwise in a credit line to the material. If material is not included in the article's Creative Commons licence and your intended use is not permitted by statutory regulation or exceeds the permitted use, you will need to obtain permission directly from the copyright holder. To view a copy of this licence, visit http://creativecommons.org/licenses/by/4.0/.

\section{References}

1. Rawlins MD, Wexler NS, Wexler AR et al (2016) The prevalence of Huntington's disease. Neuroepidemiology 46:144-153. https ://doi.org/10.1159/000443738
2. Bates G, Tabrizi S, Jones L (eds) (2014) Huntington's disease. Oxford University Press, Oxford. https://doi.org/10.1093/ med/9780199929146.001.0001

3. McColgan P, Tabrizi SJ (2018) Huntington's disease: a clinical review. Eur J Neurol 25:24-34. https://doi.org/10.1111/ene.13413

4. Carlozzi NE, Miciura A, Migliore N, Dayalu P (2014) Understanding the outcomes measures used in huntington disease pharmacological trials : a systematic review. J Huntingtons Dis 3:233-52. https://doi.org/10.3233/JHD-140115

5. Hogarth P, Kayson E, Kieburtz K et al (2005) Interrater agreement in the assessment of motor manifestations of Huntington's disease. Mov Disord 20:293-297. https://doi.org/10.1002/mds.20332

6. The Huntington Study Group (1996) Unified Huntington's disease rating scale: reliability and consistency. Mov Disord 1996(11):136-142. https://doi.org/10.1002/mds.870110204

7. Callaghan J, Stopford C, Arran N et al (2015) Reliability and factor structure of the short problem behaviors assessment for Huntington's disease (PBA-s) in the TRACK-HD and REGISTRY studies. J Neuropsychiatry Clin Neurosci 27:59-64. https://doi. org/10.1176/appi.neuropsych.13070169

8. Stout JC, Queller S, Baker KN et al (2014) HD-CAB: a cognitive assessment battery for clinical trials in Huntington's disease 1,2,3. Mov Disord 29:1281-1288. https://doi.org/10.1002/mds.25964

9. Reilmann R, Schubert R (2017) Motor outcome measures in Huntington disease clinical trials. In: Handbook of Clinical Neurology, vol 144. Elsevier B.V, pp 209-225. https://doi.org/10.1016/B9780-12-801893-4.00018-3

10. Mestre TA, Forjaz MJ, Mahlknecht P et al (2018) Rating scales for motor symptoms and signs in huntington's disease: critique and recommendations. Mov Disord Clin Pract 5:111-117. https ://doi.org/10.1002/mdc3.12571

11. Mestre TA, Busse M, Davis AM et al (2018) Rating scales and performance-based measures for assessment of functional ability in huntington's disease: critique and recommendations. Mov Disord Clin Pract 5:361-372. https://doi.org/10.1002/mdc3.12617

12. Quinn L, Busse M, Dal Bello-Haas V (2013) Management of upper extremity dysfunction in people with Parkinson disease and Huntington disease: Facilitating outcomes across the disease lifespan. J Hand Ther 26:148-155. https://doi.org/10.1016/j. jht.2012.11.001

13. Clinch SP, Busse M, Lelos MJ, Rosser AE (2018) Rethinking functional outcome measures: the development of a novel upper limb token transfer test to assess basal ganglia dysfunction. Front Neurosci. https://doi.org/10.3389/fnins.2018.00366

14. Bennasar M, Hicks YA, Clinch SP et al (2018) Automated assessment of movement impairment in Huntington's disease. IEEE Trans Neural Syst Rehabil Eng 26:2062-2069. https://doi. org/10.1109/TNSRE.2018.2868170

15. Schobel SA, Palermo G, Auinger $P$ et al (2017) Motor, cognitive, and functional declines contribute to a single progressive factor in early HD. Neurology 89:2495-2502. https://doi.org/10.1212/ WNL.0000000000004743

16. Long JD, Langbehn DR, Tabrizi SJ et al (2017) Validation of a prognostic index for Huntington's disease. Mov Disord 32:256263. https://doi.org/10.1002/mds.26838

17. Schramm C, Katsahian S, Youssov K et al (2015) How to capitalize on the retest effect in future trials on Huntington's disease. PLoS ONE 10:1-13. https://doi.org/10.1371/journal.pone.01458 42

18. Zhang Y, Long JD, Mills JA, Warner JH, Lu W, Paulsen JS, the PREDICT-HD Investigators, Coordinators of the Huntington Study Group (2011) Indexing disease progression at study entry with individuals at-risk for Huntington disease. Am J Med Genet Part B 156:751-763. https://doi.org/10.1002/ajmg.b.31232

19. Warner J, Sampaio C (2016) Modeling variability in the progression of huntington's disease a novel modeling approach applied 
to structural imaging markers from TRACK-HD. CPT Pharmacometrics Syst Pharmacol 5:437-445. https://doi.org/10.1002/ psp4.12097

20. Virtanen P, Gommers R, Oliphant TE, et al. SciPy 1.0--Fundamental Algorithms for Scientific Computing in Python. Published Online First: 23 July 2019.http://www.scipy.org/ (accessed 21 Feb 2019).

21. Wilcox R (2018) A robust nonparametric measure of effect size based on an analog of cohen's d, plus inferences about the median of the typical difference. J Mod Appl Stat Methods. https://doi. org/10.22237/jmasm/1551905677

22. Purcell NL, Goldman JG, Ouyang B, Liu Y, Bernard B, O'Keefe JA (2020) The effects of dual-task cognitive interference on gait and turning in Huntington's disease. PLoS ONE 15:e0226827. https://doi.org/10.1371/journal.pone.0226827

23. Fritz NE, Hamana K, Kelson M, Rosser A, Busse M, Quinn L (2016) Motor-cognitive dual-task deficits in individuals with early-mid stage Huntington disease. Gait Posture 49:283-289. https://doi.org/10.1016/j.gaitpost.2016.07.014

24. Tabrizi SJ, Scahill RI, Durr A et al (2011) Biological and clinical changes in premanifest and early stage Huntington's disease in the TRACK-HD study: The 12-month longitudinal analysis. Lancet Neurol 10:31-42. https://doi.org/10.1016/S1474-4422(10)70276 $-3$

\section{Authors and Affiliations}

\section{Samuel Woodgate ${ }^{1,2} \cdot$ Philippa Morgan-Jones ${ }^{1,3}$ (1) Susanne Clinch ${ }^{4} \cdot$ Cheney Drew $^{3} \cdot$ Rebecca Playle $^{3}$. Mohamed Bennasar $^{5}$ - Yulia Hicks ${ }^{1}$. Catherine Holt ${ }^{1}$ (1) Anne-Catherine Bachoud-Lévi ${ }^{6,13}$. Renaud Massart ${ }^{6,13}$.

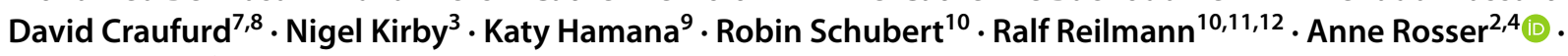 Monica Busse ${ }^{3}$ (I)}

1 Cardiff School of Engineering, Cardiff University, Cardiff, United Kingdom

2 NMRI, School of Medicine, Cardiff University, Cardiff, United Kingdom

3 Centre for Trials Research, Cardiff University, 4th Floor, Neuadd Meirionnydd, Heath Park, Cardiff CF14 4YS, United Kingdom

4 Brain Repair Group, School of Biosciences, Cardiff University, Cardiff, United Kingdom

5 School of Computing and Communications, The Open University, Milton Keynes, United Kingdom

6 Assistance Publique -Hopitaux de Paris, National Centre of Reference for Huntington's Disease, Neurology Department Henri Mondor Hospital, Creteil, France

7 Manchester Centre for Genomic Medicine, St Mary's Hospital, Manchester University NHS Foundation Trust, Manchester Academic Health Science Centre, Manchester, UK
8 Division of Evolution and Genomic Sciences, School of Biological Sciences, Faculty of Biology, Medicine and Health, University of Manchester, Manchester Academic Health Science Centre, Manchester, UK

9 School of Healthcare Sciences, Cardiff University, Cardiff, United Kingdom

10 George-Huntington-Institute, Technology-Park, Muenster, Germany

11 Department of Clinical Radiology, University of Muenster, Muenster, Germany

12 Department of Neurodegeneration and Hertie Institute for Clinical Brain Research, University of Tuebingen, Tuebingen, Germany

13 INSERM U955 01, Institut Mondor de Recherche Biomédicale, UPEC, Créteil-Ecole Normale Supérieure, PSL, Paris, France 